\title{
NOTAS
}

\section{SOBRE RESTOS DE LLEÍSMO EN MÉXICO}

Desde que en 1910 publicó Manuel Revilla sus Provincialismos de fonética en México ${ }^{1}$ nadie ha negado ni comprobado la observación, que hace al pasar, de que la $l l$ castellana sobrevive en la pronunciación de una reducida zona de México, "la Barranca de Atotonilco el Grande":

En cuanto al sonido de la $l l$ castellana, tan delicado y típico (no sé que exista en ningún otro idioma), del todo se ha perdido en América, y, por ende, en México, con excepción de algunas partes de Colombia y de una parte muy reducida de nuestro país, no lejos de Atotonilco el Grande, donde se la oye pronunciar a los campesinos con igual pureza que a los mismos asturianos $^{2}$. En el resto de nuestro territorio y de la América hispana, confúndese totalmente el sonido de la $l l$ con el de la $y$ griega ${ }^{3}$.

En esta breve afirmación convendría rectificar dos puntos: En primer lugar, un sonido idéntico al de la $l l$ castellana existe hoy en varias lenguas, entre ellas el italiano (toscano), el portugués y el catalán. En segundo lugar, no es verdad, como creyó Revilla (y siguen creyendo erróneamente la mayoría de los profesores de español), que la $l l$ se haya perdido por completo en América. La $l l$ castellana, con valor de signo distinto de la $y$, se conserva en vastas zonas de Sudamérica, a saber:

Paraguay, con las prolongaciones guaraníticas en la Argentina, Chile (menos el centro y Chiloé), y las tierras altas del viejo imperio incaico: Bolivia, Perú, Ecuador y Colombia (menos la costa). [Además,] en dos pequeñas regiones, sierra del Ecuador y Orizaba (México), se practica una distinción dentro del yeísmo: $l l=\check{z}, y=y^{4}$.

Aunque Revilla no indica su fuente de información (es decir,

${ }_{1} B A M L$, VI, 1910, págs. 368-387; estudio reproducido en el tomo IV de $B D H$, págs. 199-206.

2 "La reducida región a que aludo se denomina la Barranca de Atotonilco el Grande [en el Estado de Morelos]". $B D H$, IV, pág. 20o, n. 4. (Lo que va entre corchetes es nota de Pedro Henríquez Ureña, no del mismo Revilla).

$3 B D H$, IV, pág. 200. Los asturianos son yeístas (caye, cabayu); sólo en principio de palabra pronuncian como $l l$ - tanto la $l$-como la $l l$-castellanas (lluna, llobu; llama, llave).

4 Amado Alonso, "La $l l$ y sus alteraciones en España y América", en Estudios dedicados a Menéndez Pidal, II, pág. 69. La dualidad de $\check{z}$ y y mantenida en 
si observó el fenómeno personalmente o le llegó de segunda mano), ni dió más detalles sobre ella, su afirmación, con un pormenor geográfico añadido por primera vez en 1930 por Amado Alonso y Ángel Rosenblat, se ha venido citando desde entonces repetidamente:

También en México la Barranca de Atotonilco el Grande (Estado de Morelos) es hoy lleísta, y en esa región la única lengua indígena conocida es el náhuatl, que no tenía $l l 5$.

La 1 se conserva hasta hoy... en la Barranca de Atotonilco el Grande del Estado mexicano de Morelos ${ }^{6}$.

La $l l$... subsiste... dentro del continente septentrional, en la región de Atotonilco, del estado mejicano de Morelos 7 .

...como islote de lleísmo, se conoce uno en México, la barranca de Atotonilco el Grande, en Morelos 8 .

Dentro de ella [la región del sur de México: Oaxaca, Guerrero, Morelos] se halla el islote de $l l$ de Atotonilco el Grande ${ }^{9}$.

La $l l$ persiste en una pequeña región de México (la Barranca de Atotonilco, en Morelos) ... 10

... la distinzione fra $l l$ e $y$ originario sopravvive... in una regione ristretta del Messico ("barranca de Atotonilco el Grande", Stato di More$\operatorname{los})^{11}$.

Orizaba y la sierra ecuatoriana no puede ser en ningún sentido una distinción dentro del yeísmo, porque si la $l l$, antes de llegar a $\check{z}$, hubiera una vez pasado por la etapa y, entonces las dos y, primitiva y secundaria, para siempre confundidas, habrían corrido forzosamente la misma suerte, como sucedió, por ejemplo, en el litoral argentino: $1>y>z ̌$. Creo que la $\check{z}$ 'como fonema distinto de la y hay que explicarla por desarrollo dentro del lleismo, tal vez $1>1_{\hat{z}}>\hat{z}>\check{z}$.

Tal vez haya que rectificar el dato referente a Orizaba. La "confirmación" que recibieron Alonso y Rosenblat de un erudito natural de esa ciudad que visi taba Buenos Aires hace algunos años (Alonso, loc. cit.) puede responder a un esfuerzo cultista de distinción. No faltan en México quienes, por haber aprendido de sus maestros que cada letra tiene su propio sonido, creen que hablando correctamente deben distinguir no sólo entre la $b$ y la $v$, sino hasta entre la $s$, la $c$ y la $z$. No sería nada raro que personas cultas de Orizaba con este afán distinguidor, notando que en Puebla la $l l$ y la $y$ se confunden en $z ̌$, y en su propia región en y, creyeran que estos dos sonidos eran los que había que distinguir.

Personas avezadas a la observación lingüística me informan que en cortas visitas a Orizaba (y a Córdoba, ciudad de la misma región que tiene también fama de žeísta) no alcanzaron a oír la $\check{z}$. Por el contrario, los vendedores de helados en Orizaba gritan muy claramente ¡barquiyos! 'barquillos'. Será necesario un es. tudio más detenido sobre la pronunciación de la región para aclarar nuestra duda.

5 Alonso y Rosenblat, loc. cit.

8 Alonso y Rosenblat, $B D H$, I, pág. 195, n. 1.

7 Pedro Henríquez Ureña, $B D H$, IV, pág. 4 , n. 1.

8 Henríquez Ureña, BDH, IV, pág. 334 .

9 Henríquez Ureña, BDH, IV, pág. 340.

10 Rafael Lapesa, Historia de la lengua española, 2a ed., Madrid, 1950, pág. 33o. Pero en su mapa lingüístico del lleísmo y del voseo en América (pág. 332) aparece nuestro islote no en Morelos sino en Jalisco, por probable confusión con el Atotonilco el Alto de ese Estado.

11 Max Leopold Wagner, Lingua e dialetti dell' America Spag̀nola, Firenze, 1949, pág. 26. 
... se conserva la 1 como en Castilla en la Barranca de Atotonilco el Grande, Estado de Morelos (Revilla, l. c., luego muchas veces repetido) ${ }^{12}$.

Fuera de Colombia el sonido castellano de la $l l$ se oye en parte del estado mexicano de Morelos... 13

... at Atotonilco in Mexico $l l$ has survived where the native tongue has no such sound, but only a lengthened $l$ (as in Italian) and a click $t l^{14}$.

De la $l l$ se conserva un islote en México $\ldots{ }^{15}$, etc., etc.

Ahora bien, en México abundan los Atotonilco" ${ }^{16}$, pero según he podido comprobar consultando geógrafos ${ }^{17}$, mapas ${ }^{18}$, un censo de población ${ }^{19}$ y el mejor diccionario geográfico del territorio mexicano $^{20}$, en todo el pais el único Atotonilco que se llamaba en épocas anteriores y sigue llamándose el Grande (por existir muy cerca otro Atotonilco, llamado el Chico) está en el Estado de Hidalgo, a 30 $\mathrm{km}$. al norte de Pachuca. Y precisamente el distrito de Atotonilco el Grande está atravesado, y dividido en dos partes desiguales, por "la extensa, fragosa y pintoresca barranca de Río Grande" ${ }^{21}$. Aunque la citada barranca no tiene el nombre geográfico de "Barranca de Atotonilco el Grande", no cabe duda de que ésta es la nombrada por Revilla, porque Atotonilco el Grande es el más importante pueblo cercano, y la barranca pasa por varios kilómetros en medio del distrito del mismo nombre (único en el país). Por más señas, para los mismos habitantes de Atotonilco el Grande es simplemente "la Barranca",

12 Amado Alonso, "La $l l$ y sus alteraciones...,", art. cit., pág. 69.

${ }^{13}$ Luis Flórez, La pronunciación del español en Bogotá, pág. 240.

14 William J. Entwistle, The Spanish language, 2a ed., London, 1948, pág. 252 .

15 Berta Elena Vidal de Battini, El habla de San Luis, BDH, VII, pág. 48.

16 Atotonilco 'lugar de aguas termales' (<nahua ātl 'agua' + tōtonilli 'caliente' + co 'lugar'). Los hay (pueblos) en Durango (2), Hidalgo (2), Guanajuato (1), Morelos (1), Sinaloa (1) ; (haciendas) Durango (2), Chihuahua (1), Puebla (1), San Luis Potosí (1), Zacatecas (1); (ranchos) Durango (1), Guerrero (1), Jalisco (1), Michoacán (1), Morelos (1), Puebla (1), Veracruz (1), Zacatecas (3) ; (barrio) México (1) ; (congregaciones) Durango (1), San Luis Potosí (1) ; (minerales) Durango (1), Sinaloa (1); (rio) Chihuahua (1). Además, Atotonilquillo se llaman un pueblo, dos ranchos y una congregación de Jalisco, un rancho de Zacatecas y una hacienda de Guanajuato. Atotonilco el Alto y el Bajo los hay en Jalisco, Atotonilco el Grande y el Chico en Hidalgo. Por último, se llama Atotonilco San José una hacienda de Tlaxcala. Véase, s. v. Atotonilco, Antonio García Cubas, Diccionario geográfico, histórico y biográfico de los Es. tados Unidos Mexicanos, 5 tomos, México, D. F., 1888-1891.

17 Jorge Vivó, Rita López de Llergo y Carlos Jaso, del Instituto de Geografía de la Universidad Nacional de México.

18 Los del Instituto Panamericano de Geografía e Historia, de El Colegio de México, del Instituto de Geografía de la Universidad Nacional y del Instituto Nacional de Antropología.

19 Dirección General de Estadística, Censo de población, 1940. Los fascículos de cada Estado traen una lista completa de los pueblos, rańchos y haciendas con sus nombres oficiales y el número de sus habitantes.

20 García Cubas, op. cit.

21 García Cubas, op. cit., s. v. Atotonilco el Grande. Y hablando del pueblo de Atotonilco, cabecera de la municipalidad de su nombre, repite: "se halla situado en el llano de su nombre, que está limitado al E. por la barranca de San Sebastián o Río Grande". 
y tanto ellos como los geógrafos con quienes consulté en la Capital ${ }^{22}$, al ser preguntados por "la Barranca de Atotonilco el Grande", señalaron sin vacilación la del Río Grande o San Sebastián.

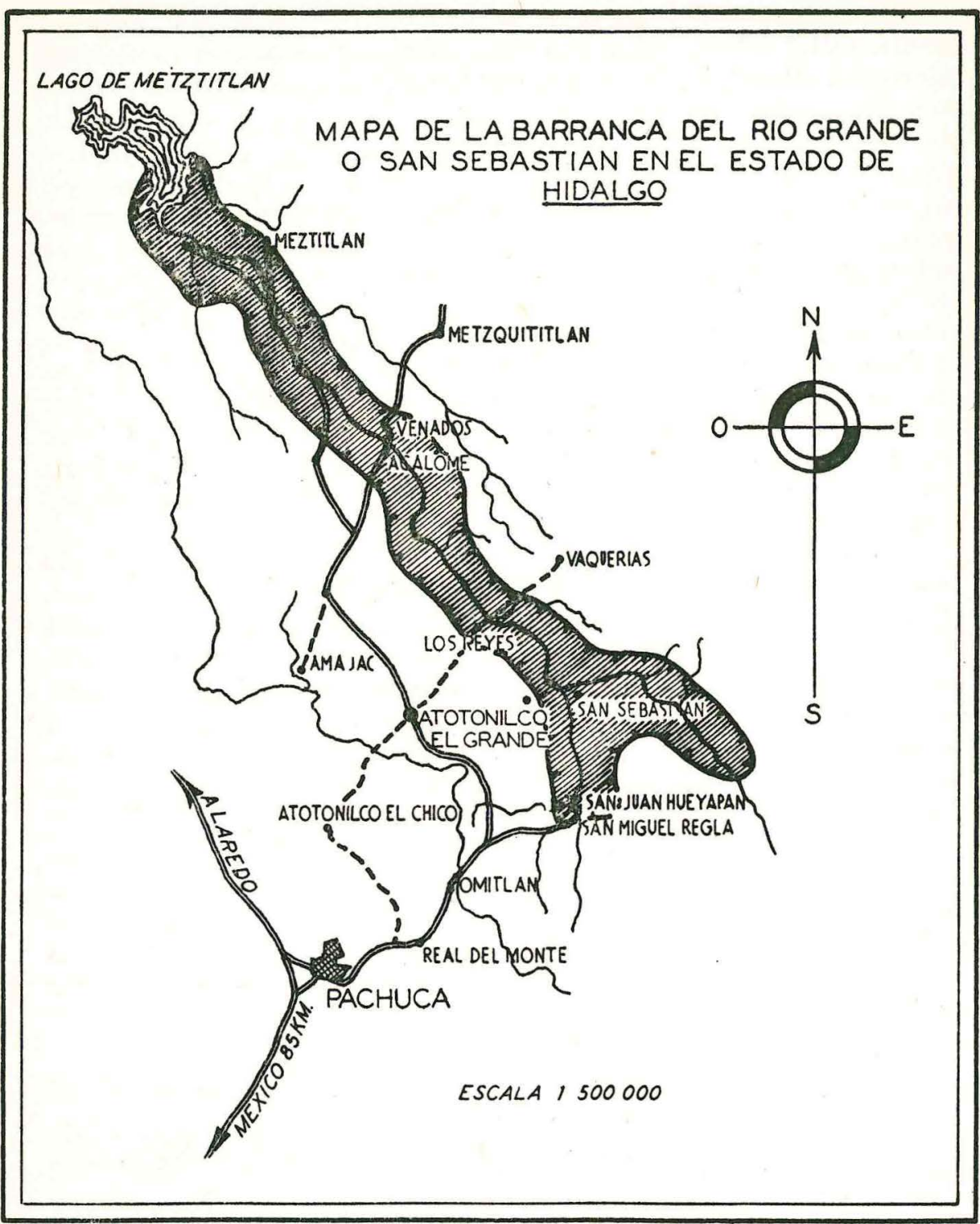

22 Uno de éstos, Carlos Jaso, es un hidalguense cuyos abuelos fueron naturales y vecinos de Atotonilco el Grande. 
Con el fin de buscar datos más concretos sobre la naturaleza, vigencia y extensión geográfico-social de esta $l l$ (si es que realmente existía), dice en este año de 1952 un viaje a Atotonilco el Grande y su barranca. Allí averigüé lo siguiente:

Si a principios del siglo pudo haber existido todavía la $l l$, lo cierto es que ya no existe ahora. No pude recorrer la barranca en toda su extensión (tiene más de $50 \mathrm{~km}$. de largo, y los escasos ranchos que hay allí se comunican sólo por una vereda que sigue la angosta orilla del río), pero comprobé que tanto los habitantes de Atotonilco el Grande como los de las haciendas de la Barranca que sí logré visi$\operatorname{tar}{ }^{23}$ son plenamente yeístas, inclusive los ancianos: yega, cabayo, yamamos, aqueyos, ayí, jayar 'hallar', payá 'para allá'. Y lo que para mí es prueba decisiva de la pérdida de la $l l$ (si existió en tiempos de Revilla) es que hasta los más familiarizados con la región, sean nativos o forasteros, desconocen en absoluto la existencia, dentro de la Barranca, de un modo de hablar distinto del suyo. De sobrevivir en cualquier parte de la Baranca un islote de personas que dijeran lama, luvia, cabalo en lugar de yama, yuvia, cabayo, sería increíble que ni siquiera los habitantes de los lugares vecinos se hubieran fijado en ello para burlarse de su manera extraña de pronunciar determinadas palabras ${ }^{24}$.

Desde 1910 la Barranca ha venido estrechando poco a poco sus relaciones con el resto del país. Aunque todavía no hay carretera que siga el curso del río, desde 1923 lo atraviesan dos en distintos sitios, con servicio de autobús, y está construyéndose otra más. También existe hoy en la Barranca una moderna planta de fuerza hidráulica. Por último la alfabetización, fuerza cultural niveladora tan notable en todo el país, no habrá dejado de influir en el habla de aquellas regiones aisladas que, como la Barranca, carecen por completo de centros propios de cultura ${ }^{25}$.

Conclusiones: 1) La "Barranca de Atotonilco el Grande", región a que aludió vagamente Revilla (1910) como único islote en la América Septentrional donde sobrevivía la $l l$ castellana, no está en el Estado de Morelos, como se ha venido afirmando desde 1930, sino en el Estado de Hidalgo. Su verdadero nombre geográfico es Barranca de Río Grande o San Sebastián.

23 Acalome, Venados, Hacienda Vieja, Buenavista, Cuesta Blanca, Carrizal, San Miguel Regla, San Juan Hueyapan, Huacatitla.

24 Por ejemplo en la Capital, sin saber nada de fonética, hay quienes se burlan de la pronunciación poblana diciendo 'Tráigame un cabažo bažo ensižado con una siža amariža'.

La $\check{z}(<l l=y)$ es característica de Oaxaca y del Valle de Puebla, incluyendo la capital del Estado, pero no se encuentra en el norte (Sierra, de Puebla), cuya población principal es Teziutlán. Véase $B D H$, IV, pág. 218 , n. 340.

${ }^{25}$ Los maestros de escuela de la Barranca vienen de regiones yeístas, y a regiones yeístas tienen que ir también los jóvenes para recibir su educación secundaria y profesional. En cambio los habitantes de la antigua ciudad colonial de Puebla se sienten orgullosos de su pretendida superioridad cultural sobre México, y, lejos de ceder al prestigio de éste, mantienen su $\check{z}$ y hasta porfían en ella. 
2) Si la $l l$ aún existía en 1910 , lo cierto es que ya no existe ahora. La región es netamente yeísta. De la $l l$ no hay el más mínimo rastro, ni siquiera entre los ancianos. Su pérdida, si ocurrió en los últimos cuarenta años, se puede explicar por el estrechamiento de lazos culturales con el resto del país debido en parte a la construcción de nuevas vías de comunicación y a la falta de un centro de cultura capaz de hacer frente al prestigio de las regiones yeístas colindantes.

Peter Boyd-Bowman

Yale University.

\section{SOBRE LA TROMPA DE PARÍS}

La más antigua referencia que conocemos hasta ahora a la "trompa de París" mencionada por Cervantes en El celoso extremeño ${ }^{1}$ se encuentra en unos versos mediocres del Bachiller Fernán Ruiz de Sevilla, autor incluído en el Cancionero de Ramón de Llavia (Sociedad de Bibliófilos Españoles, Madrid, 1945, págs. 299 y sigs.). Según el Marqués de Pidal $^{2}$ éste es uno de los primeros cancioneros que aparecen a fines del siglo xv. El poema del Bachiller se titula Una coronación de Nuestra Señora y en él se mencionan varios instrumentos musicales: unos bajo nombre equivocado ${ }^{3}$, otros, al parecer, desconocidos ${ }^{4}$. Dícese en el poema que la trompa de París es instrumento sotil, lo que resulta pertinente si el instrumento era, realmente, la guimbarda o birimbao. He aquí el fragmento donde aparece la relación de instrumentos musicales:

Jugavan de arpa e de chernubela, guitarra, xabeva, de buen añafil, de tuca bombarda, de cuarta viuela, de lira, de flauta, dulçayna gentil; laúd, monicordio, escaquer donegil, órganos, tímpano, choro, baldosa, vihuela de arco, e rota graciosa, música trompa de París sotil.

1 Véase $N R F H$, II, 1948, págs. 49 y 167 (anotaciones LII y LIII), y III, 1949, pág. 388 .

2 Citado por Emilio Pujol en su transcripción y estudio de Alonso Mudarra, Tres libros de música en cifra para vihuela, Sevilla, 1546. (Instituto Español de Musicología, Barcelona, 1949.)

3 Chernubela por churumbela; en tuca bombarda, ignoramos en qué pueda consistir el adjetivo, así como en cuarta viuela (las miges veules de Tirant lo Blanch?); escaquer por escaque (instrumento calificado de donegil, o sea, seguramente, 'doñeguil'); adrufes por adufes; bioresas por proesas (?); canón por canún; agaripe, el garip provenzal, llamado por el Arcipreste "galipe francisco".

${ }_{4}$ Palillos, ¿en el sentido actual de castañuelas, en Andalucía?; 'dulçores sería el dulcemel (?). 